\title{
Psychological Security And Self-Efficacy Among Syrian Refugee Students Inside And Outside The Camps
}

Bassam H. M. ALharbi, The Hashemite University, Jordan

\begin{abstract}
The present study aimed to identify the degree of psychological security and self-efficacy among the Syrian refugee students inside and outside the camps. The sample consisted of 600 students from Syrian refugees inside and outside the camps in the second semester of the academic year 2014-2015. Scales for psychological security and selfefficacy were implemented to answer the study questions. Results indicated low degree of psychological security for Syrian refugee students inside the camps but moderate for those outside the camps. The results also showed low degree of self-efficacy among Syrian refugee students inside and outside the camps. The results indicated also statistically significant correlation between psychological security and self-efficacy.
\end{abstract}

Keywords: Psychological Security; Self-Efficacy; Syrian Refugee

\section{INTRODUCTION}

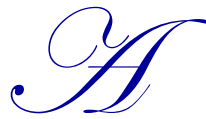

dolescents in any society show psychological stresses as a result of the nature of the developmental phase they are going through, these increasing stresses are becoming a major impact when those individuals are subject to other hard circumstances, such as immigration, asylum, and leaving homeland, where they find themselves in strange environments that they are not used to live in, and might not find enough resources to satisfy their needs and motivations, which will reflect negatively on their mental health and make them suffer from a combination of psychological stresses that include:

- Self-based stresses due to the nature of the phase in which they live

- Social-based stresses resulting from the asylum and migration conditions

In the time being, individuals in the community are subjected for many events, stresses and influences that have an impact on various aspects of their personalities, and this affect in their behaviors and emotions. Adolescents are considered to be the most affected group by these events and stresses, due to the nature of the developmental phase they are passing, which is considered to be the stage of looking forward for future life and independency and goals achieving. Adolescence is the stage of reorganizing the mental and psychological powers and all the acquired values and beliefs and experiences in childhood (Melhem 2004). Adolescence is also considered to be as a sensitive and fragile stage with feelings of anxiety and hesitation in self-concept, self-esteem, independency and self-sufficiency. In this stage, adolescents also try to have their own identity, and discover their-selves, as by the end of this stage they are in need to develop a distinctive self-image that allow them to confidently enter into the world of adults, and to have a clear idea of their personal goals and values (Stewart \& Brenano, 2006).

Ericsson indicates that the adolescent in this stage adapts with number of conflicts so that these conflicts tend to become part of his/her personality, and contribute to the sense of identity, if adolescents have a sense of confidence and feelings of independence and perseverance they look to their selves in a positive way, and feel confident about their future, which shall have a positive identity. On the other hand, fiasco at this stage can result in identity disorder, negative feeling about the self, and doubts about the future (Mousa, 2001). 
Asylum, displacement and migration are of the strongest events and stresses that have an impact on an individual's personality, perhaps for long periods of time, which negatively affect individual's activities and his/her view to the future as well as view of him/herself, as the individual is living in a country aside of his/her home, and mostly in inappropriate environments of living and studying or practicing activities amusement that these are originally found (Marina, 1998).

\section{THEORETICAL BACKGROUND}

\section{Psychological Security}

Psychological security is of the most important psychological and social needs of the individual after physiological needs and also is one of the most important motivations of human behavior, concomitant to normal psychological growth, compatibility and mental health. Psychological security in return, is closely linked to the instinct of maintain the stay on the one hand and the individual lives in saturated safe environment for his/her needs and being respected and accepted by others on the other hand (Zahran, 2003).

According to Angeliki, Leonderi, Grigoris, \& Kiosseoglon (2000) psychological security is positively correlated with the absence of feeling of fear, anxiety and inferiority, as secure individual is the one who has high levels of self-respect and esteem, and low levels of anxiety, fear and stress.

A study by Belsky \& Fearon (2002) showed that secure child has a positive perspective toward others, and this is reflected in confidence toward self and others and the world around him/her, while psychologically unsecure child in return owns negative perspective toward others, because he does not trust him/herself nor others or the world around $\operatorname{him} / \mathrm{her}$.

\section{Self-Efficacy}

The concept of self-efficacy is of the most important concepts presented by Bandura (Bandura, 1997), to show the importance of social and cognitive factors in the learning process and their interaction. According to Bandura, the individual beliefs about self-efficacy is a motive key of his/her behavior, because human behavior depends on what an individual thinks about his/her effectiveness and expectations and behavioral skills required for successful interaction with life events (Bandura, 1997).

Self-efficacy developed during childhood and adolescence, in which parents provide their children with knowledge about themselves and their abilities and the world around them, if parents provided supportive opportunities to the beliefs of the positive effectiveness as family support, and success experiences to overcome hard circumstances, this provides a solid foundation for successful adjustment and positive perception that reflects a sense of personal competence (Ciairano, Kliewer, Bonino \& Bosnia, 2008).

Self-efficacy is affected with parenting and life events; it is increased by the effectiveness of parenting level and decreased by stresses from life events (Chandler, 2006). (Barnes, 2007) indicated that self-efficacy distinguishes adolescent who have the ability to recover from the stresses more than others who are affected by stresses; because they have a strong sense of self-worth and self-esteem.

Jordan is one of the most countries in the world welcomed Syrian refugees in the Zaatari camp, the second largest refugee camp in the world, in addition to that the refugee reception was not confined to the camps, but Jordan hosts times the numbers in Jordanian cities and towns where refugees are living together with their fellow Jordanians.

Hereby, this study came to shed light on the important psychological aspects, affected by events and horrors surround the individual representing psychological security and self- efficacy, where the psychological security generated by the individual as a result of his view of what surrounds him of the circumstances and individuals, while self-efficacy generated as a result of the individual's view of self and the extent of his/her self-confidence. 


\section{LITERATURE REVIEW}

Bruce et al (2006) conducted a study to examine the relationship between parenting and stressful life events and the level of self- efficacy and depression in children ages 6-13 years. The results indicated that there is little correlation between stressful life events and self- efficacy.

Also Chung \& Elias (1996) examine the relationship between level of behavioral problems in adolescents and perceived self-efficacy and social capabilities and stressful life events. The results showed a clear correlation between behavioral problems and perceived self-efficacy and stressful life events where adolescents with high level of behavioral problems showed low level of self-efficacy and a higher level of stressful life events.

In his study about the psychological effects of the Iraqi aggression on Kuwait and children Al-Sahel (1992) conducted a study on 428 children, and found that certain disorders such as sleep disorders and phobias increased in children who lived through the Iraqi aggression against Kuwait.

Montgomery (2011) found in his study about the injury and mental health among young immigrants from the Middle East that $77 \%$ of respondents have suffered from anxiety, and $34 \%$ of them suffered from developmental disorder and mood gloomy. Similar results were found also in a study by Abdul Majeed (2004) in which abuse and psychological security among primary school students were found to be negatively correlated.

Mukhaimar (2003) also conducted a study to investigate children's perception of psychological security from parent and its relationship to anxiety and despair, the results indicated a negative correlation between the perception of psychological security by the children from parents and each of anxiety and despair, it was also found that there are differences in the perception of anxiety according to sex in favor of females.

Al-Khashali (2012) also found in a study about socializing in the Iraqi society after the US occupation that the occupation caused $90 \%$ of the children depression and sadness. Another study by Al-Ameen (2008) about the Israeli war on southern Lebanon found that children are facing difficulties to make and maintain friendships after seven months of war, and $28 \%$ of children and young people showed high levels of stress Jabali (2009) also found that behavioral problems such as aggressive behavior and distractions and excess movement and fear have appeared in children in UNRWA schools in Gaza a year after the war (2008).

And the comparative study of Abu-Nawas (2005) to compare Palestinian children living in Palestine with the refugees of their peers in the camps of Jordan, in which the results indicated that those who reside in Jordan camps they have the highest degree to avoid the traumatic event and the feeling of anguish of those who live under occupation, the results also showed that children living under occupation have registered the highest scores in these symptoms.

And the study of Worden, Gupta, and Morgos (2008). about the psychological effects of war on children's experiences in South Darfur, in which the results indicated that $75 \%$ of the children exhibit symptoms of depression and (20\%) symptoms of grief. And finally, in Germany, Silbereisen (1995) compared levels of compression factors such as divorce and moving from place of residence that increase the probability of maladaptation in early adolescence between two groups of students, one of them set the high level of compression factors and the other group a low level of stress. the sample consisted of (700) students aged (10-13 years), results indicated low level of perceived self-efficacy and an increased level of maladaptive behavior for the first group.

\section{Study Problem}

The problem of the current study is determining the psychological security and self-efficacy among students of Syrian refugees in Jordan. 
The current study is trying to answer the following questions:

1. What is the degree of psychological security among the Syrian students inside the refugee camps?

2. What is the degree of psychological security among the Syrian students outside the refugee camps?

3. What is the degree of perceived self-efficacy among Syrian students inside the refugee camps?

4. What is the degree of perceived self-efficacy among Syrian students outside the refugee camps?

5. Is there a relationship between psychological security and self-efficacy among Syrian students' refugees in camps and cities of Jordan?

\section{Significance of the Study}

The significance of the current derives from the targeted sample; the Syrian refugee students who suffer hard life events, as a result of leaving their own country, which is reflected on the psychological aspects to them.

The current study aimed to shed light on the degree of psychological security and the degree of self-efficacy among those students, which fell under the influence of forced and harsh conditions, and compared these degrees in Syrian students between who live in refugee camps, to degrees of peers of students who live in the Jordanian cities.

The findings of the current might help workers in the psychological and social aspects of refugees to have an adequate understanding of the psychological aspects they are suffering from, which facilitate putting counseling and remedial plans that can contribute to addressing these aspects.

\section{METHODOLOGY}

\section{Participants}

The study population consisted of two groups, the first group was (2647) Syrian refugee students in the eighth, ninth and tenth grade in Zaatari camp's schools, and the second group was (337) Syrian refugee students in the eighth, ninth and tenth grade outside the camps enrolled in public schools in north-west rural areas of Jordan.

The study sample for the first group was selected randomly by choosing one class for males and another for females for each grade of the eighth, ninth and tenth grades in schools Zaatari refugee camp. On the other hand, and for the second group, the researcher implemented the tools of the study on available Syrian refugee students in schools outside the camp, due to the absence of specified classes for Syrian refugee students in public schools outside the camp in the governorate of Mafraq, as Syrian refugee students are enrolled in public schools according to place of residence. The total number of respondents was (600) students as shown in Table 1.

Table 1. Demographics of Participants

\begin{tabular}{l|c|c}
\hline \multicolumn{1}{c|}{ Residence } & Number & Percentage \\
\hline Inside the refugee camp & 400 & $74 \%$ \\
\hline Outside the refugee camp & 140 & $26 \%$ \\
\hline Total & 540 & $100 \%$ \\
\hline
\end{tabular}

\section{INSTRUMENTS OF THE STUDY}

\section{Psychological Security Scale}

The scale was developed by (Maslow, 1983), it is consisted of (55) item representing positive items using 5-point Likert scale (Always $=5$, Often $=4$, Sometimes $=3$, Rarely $=2$, Never $=1$ ). This gradation might be reversed for assessing the negative aspects of the items. Total degree expected for the scale ranged 55 to 275); where high degree represents high levels of psychological security. 


\section{Checking Validity and Reliability of Instrument}

For the purpose of current study, reliability coefficient was extracted using test and re-test with time interval of two weeks on exploratory sample of 60 students from the Syrians students. The correlation coefficient for the scale was (0.82). Internal consistency (Cronbach's alpha) of the scale was (0.790).

\section{Self-Efficacy Scale}

The scale was developed by Abu Ghazal \& Alawneh (2010), it is consisted of (44) item representing positive items using 5-point Likert scale (Always $=5$, Often $=4$, Sometimes $=3$, Rarely $=2$, Never $=1$ ). This gradation might be reversed for assessing the negative aspects of the items. Total degree expected for the scale ranged (40 to 200); where high degree represents high levels of sense of self-efficacy.

\section{Checking Validity and Reliability of Instrument}

For the purpose of current study, reliability coefficient was extracted using test and re-test with time interval of two weeks on exploratory sample of 60 students from the Syrians students. The correlation coefficient for the scale was (0.83). Internal consistency (Cronbach's alpha) of the scale was (0.81).

\section{Procedures}

The researcher reached the schools to implement the instruments of the study in the classes, after clarifying the purpose of the study and its significance and presenting instructions to answer the study tools. The number of questionnaires distributed were 600, and later (50) questionnaires have been eliminated as they were not fully answered, and so the study sample was (550) students. An appropriate statistical method to answer the study questions was used.

\section{Statistical Analysis}

Means and standard deviations for each item of psychological security scale and self-efficacy scale were extracted. Bivariate Correlation was used to extract Pearson correlation coefficient to measure the strength and direction of the linear relationship (Linear Regression) between the psychological security and self-efficacy scale for Syrian student refugees in refugees camps and cities of Jordan.

\section{Results and Discussion}

What follows are results of the study according to the questions and related discussion.

First, the results regarding the first question: What is the degree of psychological security among Syrian student refugees inside the refugee camps?

To answer this question, means and standard deviations for each item of the psychological security scale from the perspective of Syrian student refugees in refugee camps was extracted, as shown in table (2).

Table 2. Mean and standard deviation for Sense of psychological security among Syrian student refugees in refugee camps

\begin{tabular}{l|c|c|c}
\hline Sense of psychological security & Mean & STD & Description \\
\hline
\end{tabular}

Table (2) shows that the mean score of the scale was (2.37) with a standard deviation of (0.215), this degree of psychological security is considered to as weak degree according to the scale of the study, as the researcher divided the expected scores of the scale to categories as follows: (5-4.2) too high, (4.19-3.4) high, (3.39-2.60) medium, (2.59-1.80) weak, less than (1.80) is unavailable. 
The results show that the rang between the highest mean and the lowest mean was (2.06), indicating proximities between the responses means of the study sample (where negative items got the least means). In return, high level of psychological security was not achieved by any item.

For the order of the means for items, table 2 shows that the highest mean was for item which state "Be sure to help my colleagues, and meeting their needs," with a mean of (3.13), while the lowest mean was for the item which state "I have painful experiences I cannot forget about it" with a mean of (1.07). looking for the values of standard deviations, we notice that they were small, and this refers to the heterogeneity in responses between the study sample.

Second, the results regarding the second question: What is the degree of psychological security among Syrian student refugees outside the refugee camps?

To answer this question, means and standard deviations for each item of the psychological security scale from the perspective of Syrian students' refugees outside refugee camps was extracted, as shown in table (3).

Table 3. Mean and standard deviation for Sense of psychological security among Syrian students' refugees outside refugee camps Sense of psychological security

\begin{tabular}{|c|c|c|}
\hline Mean & STD & Description \\
\hline 2.73 & 0.174 & Medium \\
\hline
\end{tabular}

Table (3) shows that the mean score of the scale was (2.73) with a standard deviation of (0.174), this degree of psychological security is considered to as medium degree according to the scale of the study, as the researcher divided the expected scores of the scale to categories as follows: (5-4.2) too high, (4.19-3.4) high, (3.39-2.60) medium, (2.59-1.80) weak, less than (1.80) is unavailable.

The results show that high level of psychological security was not achieved by any item in the scale. The researcher divided the expected scores of the scale to categories as follows: (5-4.2) too high, (4.19-3.4) high, (3.39-2.60) medium, (2.59-1.80) weak, less than (1.80) is unavailable. The rang between the highest mean and the lowest mean was (1.38), indicating proximities between the responses means of the study sample (where negative items got the least means). The highest mean was for item which state " I feel comfortable by my presence with people" with a mean of (3.33), while the lowest mean was for the item which state " I believe the coming days would be better " with a mean of (1.95). looking for the values of standard deviations, we notice that they were small and this refers to the heterogeneity in responses between the study sample.

Third, the results regarding the third question: What is the degree of What is the degree of perceived selfefficacy among Syrian students inside the refugee camps?

To answer this question, means and standard deviations for each item of the self-efficacy scale from the perspective of Syrian student refugees inside refugee camps was extracted, as shown in table (4).

Table 4. Mean and Standard Deviation Of Self-Efficacy Among Students Inside The Camps

Self-efficacy among students inside the camps

Mean

STD

Description

\begin{tabular}{l|l|l}
\hline 2.4 & 0.267 & Weak \\
\hline
\end{tabular}

As shown in table (4), the overall mean of the degree of self-efficacy from the standpoint of the Syrian student refugees inside the refugee camps was (2.4) with a standard deviation of $(0.267)$ which is considered to be weak degree according to the scale of the study. These results might be due to depressed conditions experienced by the Syrians students inside the camps, which reflect negatively on their perception of the extent of their effectiveness and self- efficacy. Also, the unknown and ambiguous future that waiting for them and the lack of any hope in the short term to resolve their problem increase the fears and thus the pessimistic look they look for themselves. 
The results also show that high level was not achieved by any item in the scale. The researcher divided the expected scores of the scale to categories as follows: (5-4.2) too high, (4.19-3.4) high, (3.39-2.60) medium, (2.59-1.80) weak, less than (1.80) is unavailable. The highest mean was for item which state "I have an intimate relationship with my relatives " with a mean of (3.11), while the lowest mean was for the item which state "I feel frustrated when going through a troubled health conditions " with a mean of (1.92).

Fourth, the results regarding the forth question: What is the degree of perceived self-efficacy among Syrian students outside the walls of refugee camps?

To answer this question, means and standard deviations for each item of the perceived self-efficacy scale from the perspective of Syrian student refugees outside refugee camps was extracted, as shown in table (5).

Table 5. Mean And Standard Deviation Of Self-Efficacy Among Students Outside The Camps

Self-efficacy among students outside the camps

\begin{tabular}{|l|l|l|}
\hline \multicolumn{2}{|c|}{ Mean } & \multicolumn{2}{|c|}{ STD } & Description \\
\hline 2.55 & 0.200 & Weak \\
\hline
\end{tabular}

As shown in table (5), the overall mean of the degree of self-efficacy from the standpoint of the Syrian student refugees outside the refugee camps was (2.55) with a standard deviation of $(0.200)$ which is considered to be weak degree according to the scale of the study.

The results also show that high level was not achieved by any item in the scale. The researcher divided the expected scores of the scale to categories as follows: (5-4.2) too high, (4.19-3.4) high, (3.39-2.60) medium, (2.59-1.80) weak, less than (1.80) is unavailable. The highest mean was for item which state " I think I am capable of doing anything " with a mean of (3.19), while the lowest mean was for the item which state " I can easily avoid bad situations " with a mean of (2.09).

Fifth, the results regarding the fifth question: Is there a relationship between sense of psychological security and self-efficacy among Syrian student refugees in camps and Jordanian cities?

To answer this question, Pearson correlation coefficient between scores of the study sample on psychological security scale and scores on self-efficacy scale for students' refugees in camps and Jordanian cities, the results are shown in Table 6.

Table 6. Relationship Between Sense Of Psychological Security And Self-Efficacy

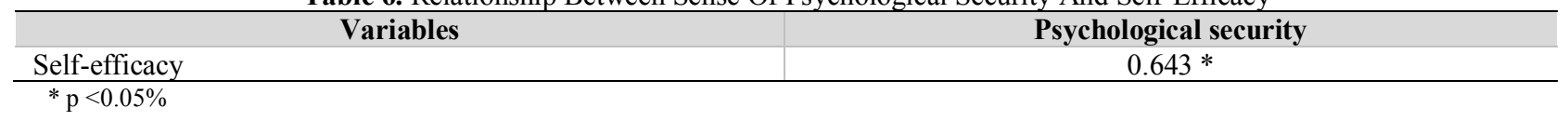

As shown in table (6), there is a strong positive significant correlation between psychological security and selfefficacy, the greater the degree of psychological security has increased the degree of self-efficacy has increased, the relationship strength was $(0.643)$, this relation was significant at $(\alpha=0.05)$.

\section{DISCUSSION}

The results of the first question might be explained as a normal result of the difficult conditions experienced by the Syrian student refugees in the camps, where the hard weather conditions, financial difficulties experienced by their families, inappropriate health care, and psychological aspects that accompanies immigration and asylum and leaving home country. These results are consisted with the results of Montgomery (2011) and Al-Khshali (2012) and Worden et al. (2008) which found that asylum and migration conditions have effects on the psychological aspects in children. 
Relating the second question, although the results of the study suggest that the degree of psychological security was within the average and not high among students outside the camps and within the Jordanian cities, but it can be attributed to the fact that Syrian students refugees in Jordanian cities outside the camps are living in conditions that are much better than the conditions experienced by peers inside camps and studying at the same public school where Jordanians students are taught, and as a result of their interaction with the citizens and the similarity of the living circumstances in which they live with others, whether in schools or houses or health centers have improved the degree of psychological security they have. This came to be as inconsistent with the results of study such Montgomery (2011) and Worden et al. (2008) which found that student refugees outside their countries are suffering from anxiety, developmental disorder, and depressed mood.

The results of the third question are inconsistent with the results of Bruce, et al (2006) about the stressful life events and the level of self-efficacy and depression in children aged of 6-13 years old, which found a weak correlation between stressful life events and self-efficacy.

But, on the other hand, the current results are consistent with the results of Chung \& Elias (1996), which indicates that there is a clear correlation between behavioral problems and perceived of self- efficacy, and between stressful life events, as adolescents with high level of behavioral problems showed lower level of self-efficacy and a higher level of stressful life events.

The results of the fourth question can be interpreted so that the Syrian students refugees in Jordanian cities and outside the camps have negative view toward themselves when comparing themselves to Jordanians students, as they are living temporarily in these cities and waiting for near or far moment to return to their countries, on the other hand, there is a huge gap in conditions and resources of the live they have compared with Jordanian citizens, that may breed a feeling of weakness and inefficiency .

These came to be consistent with results of Chung \& Elias (1996) which indicates a correlation between behavioral problems and perceived self-efficacy with stressful life events, as adolescents with high level of behavioral problems showed lower level of self-efficacy and a higher level of stressful life events.

And finally, the fifth question where its result might be explained as when individual is satisfied with sense of psychological security, lack of fear or anxiety or stress, he shows a positive look for his self-efficacy. This result came to be consistent with Silbereisen (1995) which indicates that perceived low level of self-efficacy and high level of adaptive behavior among a group of students who lived through the high levels of stress compared with a group of peers that experienced a lower level stress.

\section{RECOMMENDATIONS}

In light of the findings of the current study, the researcher recommends the following:

1. To have further studies to identify the different psychological aspects experienced by Syrian students refugees, whether inside or outside the camps.

2. To have further studies identifying the different psychological aspects experienced by students' refugees from other countries as Libya, Yemen, and Iraq, who closely share causes of migrate from their countries with different conditions they used to live in the host country.

3. The need to improve education and health environment as much as possible to those students due to their effects on the psychological side.

\section{AUTHOR BIOGRAPHIES}

Bassam H.M. ALharbi holds a Ph.D. in Psychological Counseling from the University of Jordan. He is a full-time Lecturer at The Hashemite University since 2014. 


\section{REFERENCES}

Abdul Majeed, M. (2004) Mistreatment and psychological security among a sample of primary school students. Journal of Psychological Studies, 14(22), 37-247, Cairo: issued by the Egyptian Association of psychologists.

Abu Ghazal, M. \& Alawneh, S. (2010) School justice and its relationship with self-perceived competence among a sample of elementary school students in Irbid Governorate: Evolutionary study. Damascus University Magazine, (26)4.

Abu Nuwas, J. (2005) The impact of traumatic experiences on post-traumatic stress disorder and achievement motivation and achievement at the sixth and seventh grades in Palestine and Jordan students. Unpublished MA Thesis, Amman Arab University for Graduate Studies, Amman, Jordan.

Al-Ameen, A. (2008) The psychological conditions of the children and young people in Lebanon after the July 2006 war. Beirut: Kuwait Society for the Advancement of Arab Children, vol.1

Al-Khsali, S. (2012) Socialization of children in the reality of the Iraqi society after the US occupation in 2003 - a field study in one of the Iraqi governorates (Diyala). Unpublished doctorate thesis, Open Arab Academy.

Al-Sahel, R. (1993) The social and psychological effects of the Iraqi aggression against Kuwait kids. Educational Journal, 8(26): 47-87.

Al-Saneea, S. (1999) Studies in Islamic rooting for psychology. $2^{\text {nd }}$ ed. Dar Alam Al-Koutoub, Riyadh.

Angeliki, Leonderi, Grigoris, \& Kiosseoglon (2000) The relationship of parental attachment and psychological separation to the psychological functioning of young adults. Journal of Social Psychology, 140(4), 451- 464.

Bandura, A. (1997) Self-efficacy: The exercise of control. NewYork. Freeman

Barnes, D. (2007) Divorce Transition: Identifying Risk and Promoting Resilience for Children and Their Parental Relationships. Journal of Mental and Family Therapy, 25(4), 425-441.

Belsky, J. \& Fearon, P. (2002) Early attachment security subsequent maternal sensitivity and later child development: Does continuity in development depend upon continuity of care-giving? Journal of Attachment and Human Development, 4(3), 361-387.

Bruce, A. Cole, D., Dallaries, D., Jacquez, F., Pienda, A. \& La Grage, B. (2006) Relations of parenting and negative life events to cognitive diatheses for depression in children. Journal of Abnormal Child, 34(3), 321.

Chandller, M. (2006) The influence of parenting style and ethnicity, an academic self-efficacy academic performance. A Senior Honor Thesis, Under Graduate Research Fellows, Texas University, USA.

Chung, H. \& Elias, M. (1996) Patterns of adolescent's involvement in problem behavior relationship to self- Efficacy, Social Competence, and Life Events. American Journal Of Community Psychology, 24(6), 771-784.

Ciairano, S., Kliewer, W., Bonino, S. \& Bosma, H. (2008) Parenting and adolescent well-being in two European countries. Adolescence, 47(169), 99.

Jabali, A. (2009) Behavioral problems in children after the war in Gaza and its relationship with some variables. Unpublished Master Thesis, The Islamic University, Gaza, Palestine.

Marina, A. Jdukouic (1998) Impact of displacement on the psychological well-being of refugee children. International Review of Psychiatry, 10, 186-195.

Melhem, S. (2004) Developmental psychology: Human life cycle. Dar Al-Feker Al-Arabi, Amman

Montgomery, E. (2011) Trauma, exile and mental health in young refugees. Acta Psychiatrica Scandinavica, 124(440), 1-48.

Moussa, F. (2001) Psychological development in childhood and adolescence. Al-Nahda al-Masryah Library, Cairo

Mukhaimar, E. (2003) "Kids realize the psychological security of the parent and its relationship to anxiety and despair". Journal of psychological studies, 13 (4), pp: 613-677.

Silbereisen, R. (1995) Childhood Adversities and Delinquency in Early Adolescence: Analysis of Samples from the Former Germanies. Journal of Applied Psychology, 51(2): 291-317.

Stewart, A. \& Brentano, C. (2006) Divorce: Causes and consequences. New Haven: Yale University Press.

Worden, W., and Gupta, L., \& Morgos, D. (2008) Psychosocial effects of war experiences among displaced children in Southern Darfur. Omega, 56(3), 229-53.

Zahran, H. (2003) Studies in mental health and psychological counseling. The World of Books, Cairo. 


\section{NOTES}

